\title{
Health Care Utilization and Pain Outcomes Following Early Imaging for Low Back Pain in Older Adults
}

\author{
Adam C. Powell, PhD, Teresa L. Rogstad, MPH, Sarah W. Elliott, PhD, \\ Stephen E. Price, DPT, MBA, James W. Long, BSBA, Uday U. Deshmukh, MD, MPH, \\ M. Hassan Murad, MD, MPH, and Mark W. Steffen, MD, MPH
}

Background: Professional societies have provided inconsistent guidance regarding whether older patients should receive early imaging for low back pain, in the absence of clinical indications. The study assesses the implications of early imaging by evaluating its association with downstream utilization in an elderly population.

Methods: Patients were included if they had a Medicare Advantage plan, had claims-based evidence of low back pain in 2014, and lacked conditions justifying early imaging. The outcomes examined were short-term, nonchronic, and chronic opioid use, steroid injections, and spinal surgery in the following 730 days, and persistent low back pain at 180 to 365 days. Morphine dose equivalents of opioid use was used as a measure of intensity. Logistic and $\gamma$ regressions were used to assess the association between imaging in the first 6 weeks and the outcomes.

Results: Among the 57,293 patients meeting inclusion criteria, the mean age was 71.2, and 26,606 (46.4\%) received early imaging. Early imaging was associated with increased adjusted odds of shortterm (odds ratio [OR], $1.21 ; 95 \% \mathrm{CI}, 1.15$ to 1.28$)$, nonchronic (OR, $1.78 ; 95 \% \mathrm{CI}, 1.69$ to 1.88 ), and chronic (OR, 1.13; 95\% CI, 1.07 to 1.18) opioid use, as well as steroid injections (OR, 2.55; 95\% CI, 2.28 to 2.85 ) and spinal surgery (OR, 3.40; 95\% CI, 2.97 to 3.90). Patients that received early imaging were more likely to experience persistent pain (OR, 1.09; 95\% CI, 1.05 to 1.14) and used significantly more morphine dose equivalents if they had nonchronic opioid use.

Conclusions: Early imaging for low back pain in older individuals was common, and was associated with greater utilization of downstream services and persistent pain. ( $\mathrm{J}$ Am Board Fam Med 2019;32: 773-780.)

Keywords: Diagnostic Imaging, Evidence-Based Medicine, Geriatrics, Low Back Pain, Medicare, Opioids, Outcomes Research, Pain Management, Radiology

At least 5 specialty societies have stated that physicians should not order imaging for new-onset low back pain in the absence of certain "red flags" in the

This article was externally peer reviewed.

Submitted 19 March 2019; revised 13 June 2019; accepted 13 June 2019.

From HealthHelp, Houston, TX (ACP, UUD); Humana Inc., Louisville, KY (TLR, SWE, SEP, JWL, MWS); Mayo Clinic, Rochester, MN (MHM)

Funding: Funding for the development of this manuscript was provided by HealthHelp and Humana Inc., in the form of general compensation provided to the authors. The authors were compensated for participation in research activities as a part of their job responsibilities, rather than this particular study. Dr. Murad received no funding for coauthoring this study. patient's clinical history that would warrant immediate imaging. ${ }^{1-5}$ There is some controversy regarding whether this guidance is applicable to pa-

Conflict of interest: ACP: Employment by HealthHelp and PayerProvider Syndicate. Stock ownership of Berkshire Hathaway, Community Health Systems, CVS Health Corp, HCA Healthcare, PayerProvider Syndicate, Quorum Health Corp, and Tenet Healthcare Corp. Research support from the Max Institute of Healthcare Management. TLR: Employment by Humana. SWE: Employment by Humana. SEP: Employment by Humana. JWL: Employment by Humana. UUD: Employment by HealthHelp. Stock ownership of Johnson \& Johnson, Merck, Pfizer, Express Scripts, Halyard Health Inc., Cigna, Proctor \& Gamble, and WNS Holdings. Honoraria for participation in the STOP-JIA Advisory Panel. MHM: None. MWS: Employment by Humana. 
tients over the age of 50 years. The North American Spine Society's Choosing Wisely Guideline states, "In the absence of red flags, advanced imaging within the first 6 weeks has not been found to improve outcomes," and then proceeds to list age greater than 50 years as a red flag. ${ }^{2}$ In contrast, the American College of Physicians and American Pain Society clinical practice guideline for the diagnosis and treatment of low back pain states, "For patients older than 50 years of age without other risk factors for cancer, delaying imaging while offering standard treatments and reevaluating within 1 month may also be a reasonable option." 6

Coming to a consensus regarding the imaging of older people is important because both low back pain and abnormal imaging findings have greater prevalence in older people. A study of the prevalence of low back pain found that the most afflicted population was aged 55 to 64 years $(15.4 \%)$, and the least afflicted was aged 21 to 34 years $(4.3 \%){ }^{7}$ Furthermore, the majority of older people have spinal issues; 1 cross-sectional study found that $93 \%$ of asymptomatic people aged 60 to 80 years had a degenerated disk, and $79 \%$ had a bulging disk. $^{8}$

Given the high prevalence of spinal issues even in asymptomatic older people, low back imaging has the potential to serve as a justification for more aggressive intervention, even in situations where such intervention may not be beneficial. In a general population, advanced imaging as a first management strategy for low back pain has been found to be associated with significantly increased odds of spinal surgery, epidural injections, spine surgeon visits, any spine specialist visits, and emergency department visits. ' Geographic variation in practice patterns has been shown to influence physician decision making regarding whether to pursue surgery, and higher rates of lumbar discectomy and laminectomy were found to be associated with inferior outcomes. ${ }^{10}$ A meta-analysis of 7 trials comparing imaging to no imaging for low back found a significant effect in favor of no routine imaging, in terms of severity of pain at short-term and longterm follow-up, as well as overall improvement. ${ }^{11}$

To bring additional attention to the lack of clarity that surrounds whether older individuals should

Corresponding author: Adam C. Powell, PhD, 16945 Northchase Drive, Suite 1300, Houston, Texas 77060 (E-mail: powellad@healthhelp.com). receive early imaging for new-onset low back pain, this study explored the association between early imaging and downstream utilization in a Medicare population. While this observational study is unable to show causal relationships between imaging and downstream outcomes as patients were not randomly assigned to receive imaging, its findings may inspire future researchers to conduct experiments on this topic. As America ages, the management of low back pain in older individuals will be a growing concern.

\section{Methods}

The International Classification of Diseases (ICD)-9, ICD-10, and Current Procedural Terminology (CPT) codes used to define constructs within this study are available from the corresponding author on request. They were identified by data scientists who had expertise with claims data, with periodic input from a physical therapist and multiple physicians.

\section{Data Source and Sample Population}

Claims data from January 1, 2012 through December 31, 2016 were retrospectively analyzed. The data were provided by a national health care organization and pertained to patients with Medicare Advantage health plans featuring Medicare Part D prescription drug coverage. In the United States, people age 65 years and over with eligibility for social benefits (Social Security) or meeting other criteria (eg, disability, end-stage renal disease, Amyotrophic Lateral Sclerosis) are entitled to public health insurance either through a governmentrun Traditional Medicare health plan, or through a privately run Medicare Advantage plan of their choosing, such as the one examined in this study. Patients were included in the sample if they had claims evidence of low back pain from any site of care in 2014. Low back pain was defined using the Health Effectiveness Data and Information Set definition of low back pain for the 2018 version of the Use of Imaging Studies for Low Back Pain (LBP) metric. ${ }^{12}$ Low back pain was defined using both ICD-9-CM and ICD-10-CM because it was necessary to examine patients' claims for low back pain diagnoses before and after the switch to ICD-10 on October 1, 2015.

Each patient's initial low back pain claim in 2014 served as the index event for the study. Patients were excluded from the sample if they were not 
enrolled in their health plan from 2 years before the index event to 2 years after the index event. Patients were additionally excluded if they had any claims indicating low back pain or spinal surgery in the 2 years before the index event. Specialty societies have identified a number of red flags that might justify immediate low back imaging, regardless of the duration of low back pain: history of cancer, aortic aneurysm, and progressive neurological deficits. ${ }^{6,13}$ Patients with a history of red flags as indicated in claims in the 2 years before the index event were excluded from the analysis. Codes for bladder or bowel dysfunction were used as proxies for neurological deficit. Patients with a history of trauma reported in an emergency department setting, which could signify events such as car crash injuries, were also excluded. Patients with index event claims suggesting a nonmusculoskeletal reason for low back pain (eg, calculus of the kidney, urinary tract infection, osteomyelitis, cauda equina syndrome) were excluded from the study. Lastly, exclusions were made, because of the possibility of missing data, for patients attributed to primary care physicians who delegated claims adjudication to a third party at any point from 2014 to 2016, and for patients residing in Puerto Rico or with plans specific to Puerto Rico.

\section{Measurement}

This study examined the association between the use of imaging during the first 6 weeks after the index event and the downstream care delivered, which included opioid prescription fills, morphine dose equivalents (MDEs) of opioids on hand, steroid injections, spinal surgery for low back pain, and visits in which diagnoses associated with low back pain were documented. Early imaging was defined to include any computed tomography, magnetic resonance imaging, or radiography performed on the low back during the first 6 weeks after the index event. Binary variables were created for the occurrence of opioid prescription fills at 0 to 7 days (short-term use), 8 to 90 days (nonchronic use), and 91 to 730 days (chronic use) after the index event. These breakpoints were chosen because the US Centers for Disease Control and Prevention guidelines for prescribing opioids for chronic pain state that more than 7 days of medication are rarely needed and define chronic use as use beyond 90 days. ${ }^{14}$ Count variables indicating the MDE of opioids on hand were created for the same intervals. A binary variable for epidural, facet, or any other spine-related steroid injection at 0 to 730 days after the index event was created. Patients were considered to have had spinal surgery 0 to 730 days after the index event if the claim was paired with a diagnosis code related to low back pain (using the same set of codes used to determine the index claim for low back pain). Lastly, as a measure of patient recovery, a binary variable was created for any claim featuring a diagnosis of low back pain at 180 to 365 days after the index event. The set of low back pain codes used for sample selection was used for this variable.

The study controlled for potentially confounding interventions performed during the first 6 weeks of low-back-pain management: a single physical therapy (PT) session (meant to capture superficial use of PT), more than $1 \mathrm{PT}$ session, occupational therapy, chiropractic, osteopathic manual manipulation, and unspecified chiropractic or osteopathic procedures. Use of nonnarcotic drugs for low-back-pain management during the first 6 weeks also served as a control variable. Because prior opioid use often begets future opioid use, an additional variable representing opioid use in the 90 days before the index event was included. Categorical control variables were included for the specialty of the health care provider responsible for the first claim mentioning low back pain. The health care provider could be of any specialty, with chiropractor allowed as an option. Similarly, the site of care of the health care provider producing the first claim associated with LBP was included as a control variable. Lastly, control variables were created for factors related to patients and their insurance plans: age, gender, geographic region, urbanicity, and health plan type (Health Maintenance Organization (HMO) or Preferred Provider Organization (PPO)).

\section{Outcomes and Analysis}

A series of logistic regressions were run to determine the association between the use of early imaging and each of the binary outcomes. $\gamma$ regressions were run to examine the association between early imaging and MDEs of opioid use at each of the time periods ( 0 to 7,8 to 90 , and 91 to 730 days) in the subgroups of patients with any opioid use during these periods. Both types of regression adjusted for confounders using all the control vari- 
ables. As a sensitivity analysis, analyses were rerun to see if results differed when the impact of imaging during the first 4 weeks rather than the first 6 weeks was assessed. Likewise, the analysis was rerun among the subset of patients with and without a history of opioid use in the 90 days before the index event.

\section{Results}

A total of 57,293 patients were included in the study (Figure 1). The majority of the exclusions were for a lack of continuous enrollment. Among the included patients, 26,606 (46.4\%) received imaging within 6 weeks of the onset of low back pain, and 30,687 (53.6\%) did not. As shown in Table 1, patients who received early imaging were slightly but significantly older (mean age, 71.68 vs 70.70 years), less likely to be male (35.97\% vs $39.38 \%$ ), and more likely to reside in the Southwest, Midwest, and Mid-Atlantic regions. The 2 groups did not differ significantly in the distribution of health plan types. Patients receiving early imaging were more likely to present at an outpatient facility or a hospital emergency department than in a physician's or chiropractor's office. Among patients not receiving early imaging the initial claim came most often from a primary care physician, while patients receiving early imaging were initially seen by nonprimary care specialist in the majority of cases. Opioid use during the prior 90 days did not differ between those who received early imaging and those who did not.

Early imaging was associated with significantly greater adjusted odds of all the binary outcomes examined (Table 2). The association was especially strong for steroid injections and spinal surgery, with odds ratios (ORs) exceeding 2. The OR was lowest for persistent pain. In the sensitivity analysis defining early imaging as occurring within 4 (rather than 6) weeks of pain onset, the odds ratios (not shown) were all significant and similar in magnitude to those presented for the 6-week analysis. Early imaging was significantly associated $(P<.01)$ with the intensity of nonchronic opioid use, measured in MDEs, and had a nonsignificant positive relationship with the intensity of short-term or chronic opioid use.

Among the subset of patients with a history of opioid use in the 90 days before imaging, early imaging was associated with reduced odds (OR,

Figure 1. Participant flow diagram. MAPD, medicare advantage plan that offers prescription drug coverage.




Table 1. Select Characteristics of Patients with De Novo Low Back Pain in 2014 Included in Sample

\begin{tabular}{|c|c|c|c|c|}
\hline & $\begin{array}{c}\text { Total } \\
(\mathrm{n}=57,293)\end{array}$ & $\begin{array}{c}\text { Imaging Within } \\
6 \text { Weeks of } \\
\text { LBP Onset } \\
(\mathrm{n}=26,606)\end{array}$ & $\begin{array}{c}\text { No Imaging } \\
\text { Within } 6 \text { Weeks } \\
\text { of LBP Onset } \\
(\mathrm{n}=30,687)\end{array}$ & $P$-Value ${ }^{*}$ \\
\hline Age in years (Mean, SE) & $71.20(9.55)$ & $71.68(9.38)$ & $70.70(9.70)$ & $<.001$ \\
\hline Age $<65$, n (\%) & $10,268(17.92 \%)$ & $4,302(16.16 \%)$ & $5,966(19.44 \%)$ & $<.001$ \\
\hline Age 65 to $85, \mathrm{n}(\%)$ & $43,484(75.90 \%)$ & $20,512(77.09 \%)$ & $22,972(74.85 \%)$ & \\
\hline Age $>85, \mathrm{n}(\%)$ & $3,541(6.18 \%)$ & $1,792(6.73 \%)$ & $1,749(5.69 \%)$ & \\
\hline Male, n (\%) & $21,656(37.80 \%)$ & $9,751(35.97 \%)$ & $12,085(39.38 \%)$ & $<.001$ \\
\hline Urban, n (\%) & $49,625(86.62 \%)$ & $23,148(87.00 \%)$ & $26,477(86.28 \%)$ & .011 \\
\hline Chiropractic at 6 weeks, n (\%) & $5,157(9.00 \%)$ & $1,018(3.83 \%)$ & $4,139(13.49 \%)$ & $<.001$ \\
\hline Single PT at 6 weeks, n (\%) & $1,453(2.54 \%)$ & $964(3.62 \%)$ & $489(1.59 \%)$ & $<.001$ \\
\hline Multiple PT at 6 weeks, n (\%) & $4,979(8.69 \%)$ & $2,803(10.53 \%)$ & $2,176(7.09 \%)$ & $<.001$ \\
\hline OMM at 6 weeks, n (\%) & $206(0.36 \%)$ & $64(0.24 \%)$ & $142(0.46 \%)$ & $<.001$ \\
\hline Opioid Use in prior 90 days, $\mathrm{n}(\%)$ & $14,791(25.82 \%)$ & $6,891(25.9 \%)$ & $7,900(25.74 \%)$ & .67 \\
\hline Nonnarcotic Rx at 6 weeks, $\mathrm{n}(\%)$ & $31,108(54.30 \%)$ & $16,187(60.83 \%)$ & $14,921(48.62 \%)$ & $<.001$ \\
\hline \multicolumn{5}{|l|}{ Plan type } \\
\hline HMO, n (\%) & $27,632(48.23 \%)$ & $12,910(48.52 \%)$ & $14,722(47.97 \%)$ & .19 \\
\hline LPPO, n (\%) & $15,028(26.23 \%)$ & $6,889(25.89 \%)$ & $81,39(26.52 \%)$ & \\
\hline PFFS, n (\%) & $5,111(8.92 \%)$ & $2,347(8.82 \%)$ & $2764(9 \%)$ & \\
\hline POS, n (\%) & $418(0.73 \%)$ & $182(0.68 \%)$ & $236(0.76 \%)$ & \\
\hline RPPO, n (\%) & $9,104(15.89 \%)$ & $4,278(16.07 \%)$ & $4,826(15.72 \%)$ & \\
\hline \multicolumn{5}{|l|}{ CMS region } \\
\hline $\begin{array}{l}\mathrm{AL}, \mathrm{FL}, \mathrm{GA}, \mathrm{KY}, \mathrm{MS}, \mathrm{NC}, \mathrm{SC}, \\
\mathrm{TN}, \mathrm{n}(\%)\end{array}$ & $27,232(47.53 \%)$ & $12,545(47.15 \%)$ & $14,687(47.86 \%)$ & $<.001$ \\
\hline AR, LA, NM, OK, TX, n (\%) & $9,485(16.56 \%)$ & $4,500(16.91 \%)$ & $4,985(16.24 \%)$ & \\
\hline IL, IN, MI, MN, OH, WI, n (\%) & $9,146(15.96 \%)$ & $4,321(16.24 \%)$ & $4,825(15.72 \%)$ & \\
\hline DE, DC, MD, PA, VA, WV, n (\%) & $4,422(7.72 \%)$ & $2,136(8.02 \%)$ & $2,286(7.44 \%)$ & \\
\hline Other regions, n (\%) & $7,008(12.23 \%)$ & $3,104(11.66 \%)$ & $3,904(12.72 \%)$ & \\
\hline \multicolumn{5}{|l|}{ Site of initial presentation } \\
\hline Physician office, n (\%) & $33,898(59.17 \%)$ & $13,690(51.45 \%)$ & $20,208(65.85 \%)$ & $<.001$ \\
\hline Outpatient, n (\%) & $7,539(13.16 \%)$ & $5,795(21.78 \%)$ & $1,744(5.68 \%)$ & \\
\hline Hospital ED, n (\%) & $5,525(9.64 \%)$ & $4,520(16.98 \%)$ & $1,005(3.27 \%)$ & \\
\hline Chiropractic physician, n (\%) & $4,350(7.59 \%)$ & $521(1.95 \%)$ & $3,829(12.47 \%)$ & \\
\hline Other sites, n (\%) & $5,981(10.44 \%)$ & $2,080(7.81 \%)$ & $3,901(12.71 \%)$ & \\
\hline \multicolumn{5}{|l|}{$\begin{array}{l}\text { Specialty of provider at initial } \\
\text { presentation }\end{array}$} \\
\hline Primary care, n (\%) & $26,877(46.91 \%)$ & $7,646(28.73 \%)$ & $19,231(62.66 \%)$ & $<.001$ \\
\hline Chiropractor, n (\%) & $4,384(7.65 \%)$ & $527(1.98 \%)$ & $3,857(12.56 \%)$ & \\
\hline Hospital, n (\%) & $2,942(5.14 \%)$ & $1,696(6.37 \%)$ & $1,246(4.06 \%)$ & \\
\hline Orthopaedic surgery, n (\%) & $2,763(4.82 \%)$ & $2,043(7.67 \%)$ & $720(2.34 \%)$ & \\
\hline Other or unknown specialty, n (\%) & $20,327(35.48 \%)$ & $14,694(55.22 \%)$ & $5,633(18.35 \%)$ & \\
\hline
\end{tabular}

CMS, Centers for Medicare \& Medicaid Services; ED, Emergency Department; HMO, Health Maintenance Organization; LBP, Low Back Pain; LPPO, Local Preferred Provider Organization; OMM, Osteopathic Manual Manipulation; PFFS, Private Fee-ForService; POS, Point of Service; PT, physical therapy; RPPO, Regional Preferred Provider Organization; SE, standard error.

*Significance of difference between imaging and no-imaging groups.

$0.83 ; 95 \%$ CI, 0.76 to 0.90 ) of short-term postindex opioid use, increased odds (OR, 2.36; $95 \%$ CI, 1.96 to 2.85$)$ of steroid injections, and increased odds (OR, 3.441; 95\% CI, 0.91 to 1.07 ) of spinal surgery. No association of early imaging with other outcomes was detected: neither nonchronic (OR, 1.07;
95\% CI, 0.98 to 1.16 ) or chronic (OR, $0.95 ; 95 \%$ CI, 0.88 to 1.03$)$ opioid use, or pain at 180 to 365 days (OR, 0.99; 95\% CI, 0.91 to 1.07 ). In contrast, results for opioid-naïve patients were in line with those for the overall population. Among the opioid-naïve, early imaging was associated with in- 
Table 2. Adjusted* Odds Ratios (ORs), Imaging in the First 6 Weeks vs. No Imaging in the First 6 Weeks for Patients with De Novo Low Back Pain in 2014

\begin{tabular}{lll}
\hline Dependent Variable & OR & $95 \%$ CI \\
\hline Short-term opioid use & 1.21 & 1.15 to 1.28 \\
Nonchronic opioid use & 1.78 & 1.69 to 1.88 \\
Chronic opioid use & 1.13 & 1.07 to 1.18 \\
Steroid injections & 2.55 & 2.28 to 2.85 \\
Spinal surgery & 3.40 & 2.97 to 3.90 \\
Persistent low back pain & 1.09 & 1.05 to 1.14 \\
\hline
\end{tabular}

CI, confidence interval.

*Adjustment factors were interventions in the first 6 weeks after the index date (single physical therapy visit, multiple physical therapy visits, chiropractic visit, osteopathic visit, nonnarcotic prescription for pain relief), use of opioids in the prior 90 days, specialty of specialist at initial presentation, site of care at initial presentation, age, sex, region, urbanicity, and health plan type.

creased odds of short-term (OR, 1.53; 95\% CI, 1.43 to 1.63 ), nonchronic (OR, 2.38; 95\% CI, 2.24 to 2.54 ), and chronic (OR, 1.27; 95\% CI, 1.19 to 1.36) postindex opioid use, increased odds of steroid injections (OR, 2.61; 95\% CI, 2.28 to 3.00), increased odds of spinal surgery (OR, 3.33; $95 \%$ CI, 2.83 to 3.93), and increased odds of pain at 180 to 365 days (OR, $1.15 ; 95 \% \mathrm{CI}, 1.09$ to 1.21 ).

\section{Discussion}

In a population of patients with Medicare Advantage insurance, early imaging was associated with increased downstream utilization of all the services examined, as well as reduced likelihood of pain resolution at 180 to 365 days. These findings are consistent with prior findings pertaining to younger populations. ${ }^{9,11}$ Results lend support to the American College of Physicians and American Pain Society's guideline advising against pursuing early imaging solely based on a patient's age. The association between imaging and spinal surgery has been found by prior research. ${ }^{15}$ Furthermore, an observational study found that $22 \%$ of the regional variability in spine surgery rates can be accounted for by variability in the rates of Computed Tomography (CT) and Magnetic Resonance Imaging (MRI) of the spine. ${ }^{16}$

There are several possible explanations for the findings of this study. When early imaging is performed in patients without conditions that are recognized as justification for imaging, the physician may be pursuing a more aggressive diagnostic strat- egy. If such physicians were likewise inclined to pursue aggressive treatment strategies, our findings would be observed. A similar result would occur if some patients pressured their physicians to pursue both diagnosis and treatment aggressively. Alternatively, patients with more symptoms might be more likely to undergo more intensive diagnostic workup as well as more intensive treatment.

At the population level, increased low-back imaging has occurred alongside increased low-back interventions. An analysis of Traditional Medicare data found that from the mid-1990s to the early2000s, there was an upward trend in lumbar spine MRI, contemporaneous with upward trends in opioid analgesic prescriptions for spine problems, lumbosacral injections, and lumbar fusions for degenerative spine conditions. ${ }^{17}$ Contemporary data from the United States Medical Expenditure Panel Survey, pertaining to the general adult population, showed that these increases in utilization were paired with increases in the rates of self-reported functional limitations, mental health, work limitations, and social limitations among people reporting spine problems. ${ }^{18}$

Although there are multiple possible explanations for the findings, the consistency of the statistically significant associations across all measured outcomes is potentially cause for concern. The magnitude of the association of early imaging with steroid injections (OR, 2.55) and spinal surgery $(\mathrm{OR}, 3.40)$ is particularly noteworthy. While patients with early imaging received more downstream interventions, they were no less likely to have evidence of continuing back pain at 180 to 365 days. Findings of this study are consistent with those of a meta-analysis of 7 prior trials, which found that patients receiving diagnostic imaging did not experience improved outcomes at 1-year followup. ${ }^{11}$ To the extent that greater use of the measured therapies was unnecessary, early imaging may increase patients' exposure to invasive procedures and contribute to the national opioid crisis that has been declared a public health emergency by the US Department of Health \& Human Services. ${ }^{19}$

In light of the opioid crisis, a secondary analysis was conducted to assess the differential impact of early imaging on opioid-naïve patients and patients with a history of opioid use in the prior 90 days. Although early imaging was significantly associated with chronic postindex opioid use in opioid-naïve 
patients in the overall study group, this association was not observed in patients with a history of use. Future researchers may wish to more directly explore the differential impact of imaging on opioidnaïve patients versus patients with previous opioid use.

\section{Limitations}

There are a number of potential limitations to the analysis. Patients were not randomly assigned to early imaging, and there were baseline demographic differences between the groups that did and did not undergo early imaging (Table 1). While the 2 groups did not significantly differ in their rates of prior opioid use, patients with early imaging had significantly more intense use of several treatments in the first 6 weeks: PT, osteopathic manual manipulation, and nonnarcotic prescription drugs. Regression models controlled for these differences, but unmeasured confounders may have influenced results.

It was not possible for this study to control for symptom severity, which is not captured in claims data. It is possible that there was confounding, with patients experiencing greater pain receiving both more early imaging and more interventions. However, the variables controlling for early treatments (eg, PT) may be an indirect control for severity since patients in more intense pain may have pursued more early treatments.

The findings of the study may not be generalizable to other populations, as the sample included a primarily Medicare Advantage population that was unevenly distributed across the United States (Table 1). The proportion of patients receiving lowback imaging in the absence of red flags, $46.4 \%$, was higher than the $22.5 \%$ reported by a 2006 to 2011 analysis of Traditional Medicare claims ${ }^{20}$; the discrepancy may be attributable to differences in populations, time frame, and methods for identifying low back pain. Geography has been shown to play a strong role in the rate of spinal CT and MRI imaging; the Hospital Referral Regions with the highest rates perform over 7 times more CT and MRI imaging as the Hospital Referral Regions with the lowest rates. ${ }^{16}$ As the data pertain to patients with an initial low-back-pain claim in 2014, and the declaration of a public health emergency related to opioid use was not made until 2017, it is possible that the patterns of opioid utilization shown in the study are different from those that would be seen today. ${ }^{19}$

Finally, there were imperfections in the way that patients with red flags or a history of low back pain were excluded. It is possible that patients may have had a history of low back pain or red flags based on events further in the past than the 2-year look-back period. Some red flags may have been missed because of the limitations of claims data. Most notably, as only codes for bladder and bowel dysfunction were used as proxies for progressive neurological deficits warranting early imaging, some appropriate exclusions may have been missed.

\section{Conclusions}

Early low-back imaging of older patients was associated with more intensive downstream interventions, including greater use of opioids and invasive procedures, without claims evidence of improvement in pain relief. The observational findings of this study suggest the need for further research to determine whether the caution around early imaging for patients aged 50 and younger should be extended to patients of all ages across all guidelines. A randomized controlled trial of the impact of early imaging on older patients could definitively settle this question.

\section{Implications}

Efforts should be made to inform clinicians and patients of the debatable value of early imaging in the absence of comorbidities. Professional societies that place age limitations on their guidance against early imaging for low back pain should consider revisiting the evidence behind their age limitations.

The authors thank Stephenia Scott, Kevin Wells, and Jennifer Megibben for their contributions to the development of the dataset for this study.

To see this article online, please go to: http://jabfm.org/content/ 32/6/773. full.

\section{References}

1. ACP_Avoid imaging for low back pain । Choosing Wisely. 2012. Available from: http://www.choosing wisely.org/clinician-lists/american-college-physiciansimaging-for-non-specific-low-back-pain/. Accessed January 8, 2019.

2. NASS_-Imaging for low back pain I Choosing Wisely. 2013. Available from: http://www.choosingwisely.org/ clinician-lists/north-american-spine-society-advanced- 
imaging-of-spine-within-first-six-weeks-of-non-specificacute-low-back-pain/. Accessed December 7, 2018.

3. AAFP_Imaging for low back pain । Choosing Wisely. 2012. Available from: http://www.choosingwisely.org/ clinician-lists/american-academy-family-physiciansimaging-low-back-pain/. Accessed January 8, 2019.

4. AANS-Imaging of spine for acute low back pain Choosing Wisely. 2014. Available from: http://www. choosingwisely.org/clinician-lists/american-associationneurological-surgeons-imaging-for-nonspecific-acutelow-back-pain/. Accessed January 8, 2019.

5. ACOEM-X-rays for acute non-specific low back pain I Choosing Wisely. 2014. Available from: http:// www.choosingwisely.org/clinician-lists/americancollege-occupational-environmental-medicine- $\mathrm{x}$-raysfor-acute-non-specific-low-back-pain/. Accessed January 8, 2019.

6. Chou R, Qaseem A, Snow V, et al. Diagnosis and treatment of low back pain: a joint clinical practice guideline from the American College of Physicians and the American Pain Society. Ann Intern Med 2007;147:478-491.

7. Freburger JK, Holmes GM, Agans RP, et al. The rising prevalence of chronic low back pain. Arch Intern Med 2009;169:251-258.

8. Boden SD, McCowin PR, Davis DO, Dina TS, Mark AS, Wiesel S. Abnormal magnetic-resonance scans of the cervical spine in asymptomatic subjects. A prospective investigation. J Bone Joint Surg Am 1990;72:1178-1184.

9. Fritz JM, Brennan GP, Hunter SJ. Physical therapy or advanced imaging as first management strategy following a new consultation for low back pain in primary care: associations with future health care utilization and charges. Health Serv Res 2015;50: 1927-1940.

10. Keller RB, Atlas SJ, Soule DN, Singer DE, Deyo RA. Relationship between rates and outcomes of operative treatment for lumbar disc herniation and spinal stenosis. JBJS 1999;81:752.

11. Karel YH, Verkerk K, Endenburg S, Metselaar S, Verhagen AP. Effect of routine diagnostic imaging for patients with musculoskeletal disorders: a metaanalysis. Eur J Intern Med 2015;26:585-595.

12. HEDIS Measures and Technical ResourcesNCQA. 2018 Available from: https://www.ncqa.org/ hedis/measures/. Accessed December 7, 2018.

13. Choosing wisely: avoid imaging studies (MRI, CT or X-rays) for acute low back pain without specific indications. Am Fam Physician. Available from: https://www. aafp.org/afp/recommendations/viewRecommendation. htm? recommendationId=180. Accessed March 1, 2019.

14. Dowell D, Haegerich TM, Chou R. CDC guideline for prescribing opioids for chronic pain-United States, 2016. JAMA 2016;315:1624-1645.

15. Verrilli D, Welch HG. The impact of diagnostic testing on therapeutic interventions. JAMA 1996; 275:1189-1191.

16. Lurie JD, Birkmeyer NJ, Weinstein JN. Rates of advanced spinal imaging and spine surgery. Spine 2003;28:616-620.

17. Deyo RA, Mirza SK, Turner JA, Martin BI. Overtreating chronic back pain: time to back off? J Am Board Fam Med 2009;22:62-68.

18. Martin BI, Deyo RA, Mirza SK, et al. Expenditures and health status among adults with back and neck problems. JAMA 2008;299:656-664.

19. PHE. Determination that a public health emergency exists. 2018. Available from: https://www.phe.gov/ emergency/news/healthactions/phe/Pages/opioids. aspx. Accessed December 7, 2018.

20. Colla CH, Morden NE, Sequist TD, Schpero WL, Rosenthal MB. Choosing wisely: prevalence and correlates of low-value health care services in the United States. J Gen Intern Med 2015;30:221-228. 